\title{
Soluble interleukin 2 receptors in patients with polymyositis/dermatomyositis
}

\author{
Yoshiaki Tokano, Yoshinori Kanai, Hiroshi Hashimoto, Ko Okumura, Shun-ichi Hirose
}

\begin{abstract}
The concentration of soluble interleukin 2 receptor was determined in serum samples from 19 patients with polymyositis/dermatomyositis by an enzyme linked immunosorbent assay (ELISA). The concentration of soluble interleukin 2 receptor in serum samples from patients with polymyositis/dermatomyositis was higher than that in samples from normal subjects.
\end{abstract}

The 55 kilodalton chain of human interleukin 2 receptor is released in a soluble form from activated $\mathrm{T}$ cells. Soluble interleukin 2 receptor is detected in vitro in the supernatant of $T$ cell cultures activated by mitogens or antigens. In vivo, high concentrations of soluble interleukin 2 receptor have been reported in patients with leukaemia or malignant lymphoma, in transplant recipients, and in patients with various autoimmune diseases. In patients with rheumatic diseases, high concentrations of soluble interleukin 2 receptor have been reported in patients with rheumatoid arthritis (RA), ${ }^{1-4}$ systemic lupus erythematosus (SLE), ${ }^{56}$ and systemic sclerosis. ${ }^{7}$ In these diseases, the concentration of soluble interleukin 2 receptor has been shown to correlate with other indicators of disease activity.

In this study, we determined the concentration of soluble interleukin 2 receptor in serum samples from patients with polymyositis/ dermatomyositis for the examination of activated $\mathrm{T}$ cells.

\section{Patients and methods}

Serum samples were obtained from 19 patients with polymyositis/dermatomyositis ( 15 women,

Division of

Rheumatology,

Department of

Internal Medicine,

Juntendo University,

School of Medicine,

2-1-1, Hongo,

Bunkyo-ku, Tokyo 113,

Japan

Y Kanai

H Hashimoto

$S$ Hirose

Department of

Immunology,

Juntendo University,

School of Medicine,

2-1-1, Hongo,

Bunkyo-ku, Tokyo 113, Japan

K Okumura

Correspondence to: Dr Tokano.

Accepted for publication 12 November 1991 four men, aged 28-48 years), according to Bohan's criteria, ${ }^{8}$ and from 20 normal subjects matched for age and sex. Eleven patients had dermatomyositis and eight patients had polymyositis. The severity of serological indices and clinical symptoms varied between patients. All but four patients had been receiving treatment with steroids (30-50 mg/day prednisolone) for one to six months.

The concentration of soluble interleukin 2 receptor was determined using a commercial kit (T Cell Science, Cambridge, MA, USA) as described previously. ${ }^{6}$ This kit is a sandwich enzyme linked immunosorbent assay (ELISA) for the detection of soluble interleukin 2 receptor in human serum samples. After the beads coated with monoclonal antibodies to interleukin 2 receptor had been placed in the polyethylene tubes, $200 \mu \mathrm{l}$ serum sample and $200 \mu \mathrm{l}$ peroxidase conjugated monoclonal anti- body to interleukin 2 receptor directed against a second epitope on the interleukin 2 receptor molecule were added and the tubes were incubated at room temperature for 90 minutes. After washing several times, $o$-phenylenediamine (in $\mathrm{pH} 5.0$ citrate buffer and hydrogen peroxide) was added and the sample was left to stand for 30 minutes at room temperature. Finally, 2.5 M sulphuric acid was added to terminate the reaction and the absorbance was determined at $490 \mathrm{~nm}$. The concentration of soluble interleukin 2 receptor in the serum samples was determined by a reference curve generated from a set of standards.

The statistical significance of the difference in concentration of soluble interleukin 2 receptor between the patients with polymyositis/ dermatomyositis and normal subjects was obtained using Welch's $t$ test.

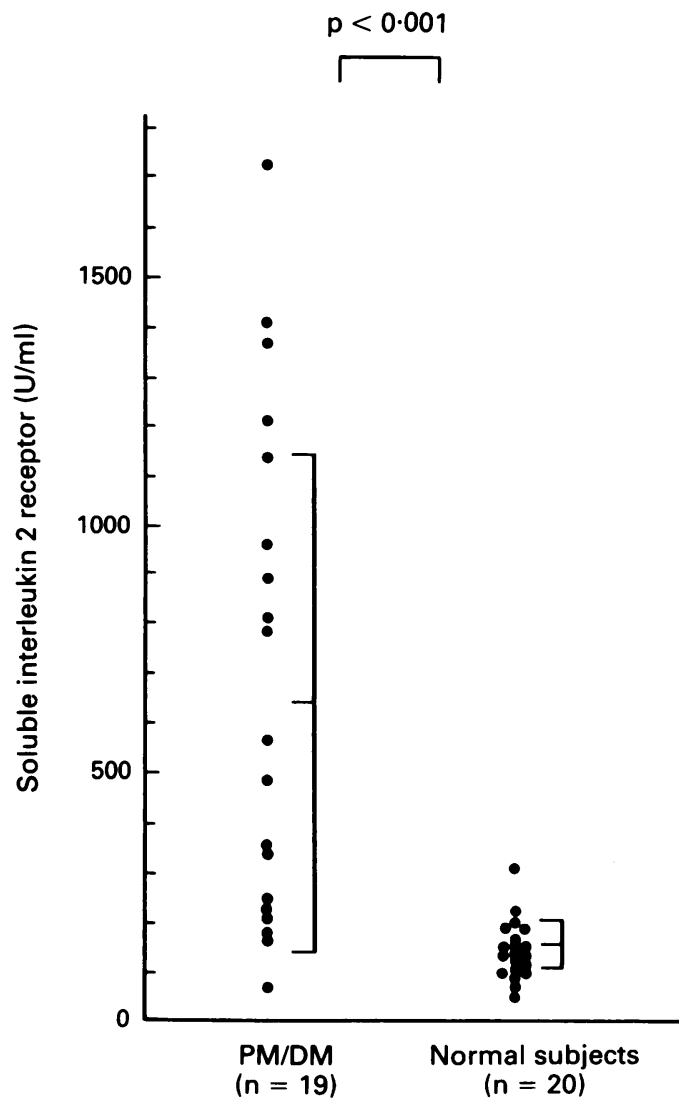

Concentration of soluble interleukin 2 receptor in serum samples from 19 patients with polymyositis/dermatomyositis $(P M / D M)$ and 20 normal control subjects. The

concentrations of soluble interleukin 2 receptor are plotted in $\mathrm{U} / \mathrm{ml}$; the unit values were determined by a reference curve generated from standard solutions (see text). The central horizontal bar indicates the mean value and the upper and lower horizontal bars the standard deviation. The statistical significance was determined with Welch's t test. 


\section{Results and discussion}

The figure shows the concentrations of soluble interleukin 2 receptor, assessed by the ELISA, in serum samples from 19 patients with polymyositis/dermatomyositis and 20 normal subjects. The patients with polymyositis/ dermatomyositis had significantly higher concentrations of soluble interleukin 2 receptor, ranging from 75 to $1729.7 \mathrm{U} / \mathrm{ml}$ (mean (SD) $638 \cdot 2(506 \cdot 1) \mathrm{U} / \mathrm{ml})$, than the normal subjects, whose range was between 90 and $310 \mathrm{U} / \mathrm{ml}$ $(155.05(48.77) \mathrm{U} / \mathrm{ml})(\mathrm{p}<0.001)$. The four patients who were not receiving treatment with steroids also had higher concentrations of soluble interleukin 2 receptor $(1208,1363,785$, and $1140 \mathrm{U} / \mathrm{ml}$ ).

Soluble interleukin 2 receptor is detected in culture supernatants or in serum samples during $T$ cell proliferation. It has therefore been considered that it is a marker of $T$ cell malignancy or activated T cells. High concentrations of soluble interleukin 2 receptor have been reported in $T$ cell malignancies (adult $T$ cell leukaemia and Sézary's syndrome), other leukaemias or lymphomas (B cell chronic lymphocytic leukaemia, hairy cell leukaemia, acute lymphocytic leukaemia, Hodgkin's disease, and non-Hodgkin's lymphoma), chronic infections (bacterial endocarditis and leprosy), acquired immune deficiency syndrome, sarcoidosis, and renal or cardiac allograft recipients. In rheumatic diseases, previous studies have reported that patients with $\mathrm{RA},{ }^{1-4}$ SLE, ${ }^{56}$ and systemic sclerosis ${ }^{7}$ have high concentrations of soluble interleukin 2 receptor. It is considered that high concentrations of soluble interleukin 2 receptor in these patients are related to the activated $T$ cells. The concentrations of soluble interleukin 2 receptor have not been examined in other rheumatic diseases, however.

In this study, we determined the concentration of soluble interleukin 2 receptor in serum samples from patients with polymyositis/ dermatomyositis. It was found that the concentrations of soluble interleukin 2 receptor in patients with polymyositis/dermatomyositis were significantly higher than those of normal subjects. This suggests that patients with polymyositis/dermatomyositis also have activated T cells, and supports previous work. ${ }^{9}$ Although it has been considered that activated $T$ cells only occur in patients with SLE and RA, it was found that they also occur in other rheumatic diseases. As soluble interleukin 2 receptor can more easily detect activated $T$ cells than other markers (such as HLA-DR positive $T$ cells), it is suggested that soluble interleukin 2 receptor could be a useful marker of activated $T$ cells in various rheumatic diseases.

1 Symons J A, Wood N C, Di Giovine F S, Duff G W. Soluble IL-2 receptor in rheumatoid arthritis. Correlation with disease activity, IL-1 and IL-2 inhibition. F Immunol 1988 , 141: 2612-8.

2 Keystone E C, Snow K M, Bombardier C, Chang C $\mathbf{H}$, Nelson D L, Rubin L A. Elevated soluble interleukin-2 receptor levels in the sera and synovial fluids of patients receptor levels in the sera and synovial fluids of patients
with rheumatoid arthritis. Arthritis Rheum 1988; 31:844-9.

with rheumatoid arthritis. Arthrits Rheum 1988; 31: 844-9.

G R, Martin W J. Serum levels of interleukin-2 receptor and activity of rheumatic disease characterized by immune system activation. Arthritis Rheum 1988; 31: 1358-64.

4 Wood N C, Symons J A, Duff G W. Serum interleukin-2receptor in rheumatoid arthritis: a prognostic indicator of disease activity? I Autoimmun 1988; 1: 353-61.

5 Wolf R E, Brelsford W G. Soluble interleukin-2 receptors in systemic lupus erythematosus. Arthritis Rheum 1988; 31 : 729-35.

6 Tokano Y, Murashima A, Takasaki Y, Hashimoto H, Okumura K, Hirose S. Relation between soluble interleukin-2 receptor and clinical findings in patients with systemic lupus erythematosus. Ann Rheum Dis 1989; 48: 8ystem 803 .

7 Degiannis D, Seibold J R, Raskova J, Raska K, Czarnecki M Soluble interleukin-2 receptors in patients with systemic sclerosis. Arthritis Rheum 1990; 33: 375-80.

8 Bohan A, Peter J B. Polymyositis and dermatomyositis. New Engl f Med 1975; 292: 344-7.

9 Miller F W, Love L A, Barbieri S A, Balow J E, Plot\% P H. Lymphocyte activation markers in idiopathic myositis: changes with disease activity and differences among clinical and autoantibody subgroups. Clin Exp Immunol 1990; 81 . 373-9. 